\title{
LA INVESTIGACIÓN DE LAS CIENCIAS ECONÓMICAS, ADMINISTRATIVAS Y CONTABLES EN LA EDUCACIÓN SUPERIOR DE HONDURAS
}

Manuel Antonio Flores Fonseca, Universidad Nacional Autónoma de Honduras, Instituto de Investigaciones Económicas y Sociales (IIES UNAH), Ciudad universitaria edifico c2 primer piso. Tel./Fax: 504-22391849 Correo electrónico: mflores@iies-unah.org

\section{RESUMEN}

La investigación de la educación superior en el país está concentrada en las instituciones públicas. La Universidad Nacional Autónoma de Honduras (UNAH), pública, de mayor data y presencia nacional, cuenta con 6 institutos de investigación (económicas, jurídicas, ciencias de la tierra, microbiología, paz y democracia, derechos humanos), 86 investigadores y 14 unidades de investigación. La Universidad Pedagógica Nacional Francisco Morazán (UPNFM) cuenta con un Instituto de Investigación y Evaluación Educativa. Otras instituciones públicas como la Universidad Nacional de Agricultura (UNA) y la Escuela Nacional de Ciencias Forestales (ESNACIFOR) han fomentado la investigación agrícola y forestal respectivamente. En los centros privados, la Universidad El Zamorano, ha desarrollado una vasta carrera investigativa en el campo agrícola. En el ranking iberoamericano de la actividad investigadora solo 7 de 20 Instituciones de Educación Superior hondureña aparecen.

El objetivo de este trabajo es presentar el estado de la investigación de las ciencias económicas, administrativas y contables en las instituciones de educación superior del país. Detallar la oferta de centros, las líneas o programas, proyectos, investigadores, publicaciones a través de libros, manuales y documentos, artículos científicos en revistas indexadas, la enseñanza de la investigación en los pensum de los grados académicos, programas o proyectos de investigación vinculados con la empresa y gobierno. La metodología de este trabajo incluye una revisión bibliográfica documental de información de la educación superior, específicamente de la relativa a las ciencias económicas, administrativas y contables. Una recopilación y revisión de información de las líneas y proyectos de investigación, publicaciones, investigadores, programas de estudio, proyectos de vinculación en las facultades, escuelas e institutos del área específica.

Palabras Clave: Investigación; Educación Superior; Ciencias Económicas Administrativas y Contables 


\title{
RESEARCH INTO ECONOMICS SCIENCE, MANAGEMENT AND ACCOUNTING IN EDUCATION HIGHER HONDURAS
}

\author{
Manuel Antonio Flores Fonseca, Universidad Nacional Autónoma de Honduras, \\ Instituto de Investigaciones Económicas y Sociales (IIES UNAH), \\ Ciudad universitaria edifico c2 primer piso. Tel./Fax: 504-22391849 \\ Correo electrónico: mflores@iies-unah.org
}

\begin{abstract}
Research of higher education in Honduras is focused on public institutions. The National Autonomous University of Honduras (UNAH), which is public and of upmost and longest presence, has 6 research institutions (which include economics, laws, earth science, microbiology, peace and democracy, and human rights), 86 researchers and 14 units of investigation. The National Pedagogical University Francisco Morazán (UPNFM) has an Institute of Educational Research and Evaluation. Other public institutions such as the National Agricultural University (UNA) and the National School of Forest Sciences (ESCANIFOR) have promoted agricultural and forest investigations respectively. Concerning private centers, the El Zamorano University has developed a vast investigative career in the agricultural field. The Latin American Ranking of Research Activity includes only 7 out of 20 institutions of higher education in Honduras.
\end{abstract}

The following work presents the current state of the investigation of economic, administrative, and accounting sciences in higher education institutions in the country. Also, to detail the academic offer of such centers, the programs, projects, researchers, publications through books, manuals and documents, scientific articles on indexed magazines, research education in pensum of academic degrees, programs or research projects involved with the government or private companies. The methodology of this work includes a documented bibliographic revision of information related to higher education, specifically related to economic, administrative, and accounting sciences. A compilation and an informational revision of the alignments and investigation projects, publications, researchers study programs, faculty projects, schools and institutions of specific areas.

Keywords: Research; Higher Education; Economics, Administratives and Contables Sciences. 


\section{INTRODUCCIÓN.}

Las tendencias mundiales de la educación superior apuntan a un crecimiento general sin precedentes, 152,5 millones en 2007, con una tendencia a la feminización que ha superado la mitad del total y donde las Ciencias Sociales, Educación Comercial y Derecho se convierten los campos del conocimiento mayormente seleccionado por los estudiantes.

América Latina también vive la masificación de la educación superior, ha pasado de 7 millones a 18 millones de estudiantes matriculados (1994-2008). La demanda de estudios de posgrado se ha incrementado principalmente por el alza en las ciencias sociales y administrativas, el área más grande de todas, que comprende administración de empresas, leyes, psicología, economía y ciencias sociales. Estas tendencias se han mantenido en los últimos 10 años, porque los sistemas de posgrado favorecen programas de maestría concentrados en las ciencias sociales y administrativas (CEPAL, 2009). El mayor número de investigadores con los que cuenta la región, se ubican mayoritariamente en las instituciones de educación superior, sobre todo en las universidades públicas: $65,1 \%$ del total.

En términos de las publicaciones científicas, América Latina y el Caribe cubren tan solo el $2.6 \%$ del total de las publicaciones a nivel mundial.

La educación superior hondureña en la última década ha alcanzado los niveles más grandes de su historia, en dieciséis años se incrementó más de tres veces, actualmente tiene 173 mil estudiantes. La modalidad presencial es la preponderante, la virtual ha sido insignificante e incluso ha desaparecido del sistema y la de distancia aunque aumentó su número y proporción, supera apenas un cuarto del total. Prima la feminización de los estudiantes, principalmente en las dos más grandes instituciones públicas. La oferta educativa de educación superior hasta el 2008 es de 349 grados, de los cuales la mitad es ofrecida por instituciones públicas. El 55\% de la oferta de grados es ofrecida a nivel de licenciatura. En las áreas del conocimiento en el 2010 hay una alta concentración de la oferta educativa en el sector de ciencias sociales, ciencias comerciales y derecho, que sumadas a la enseñanza de la educación y humanidades alcanza el 66\%. La cobertura educativa superior del país es baja, apenas alcanza el 15.7\% y su avance a través del tiempo ha sido lento (Flores, 2012). 


\section{OBJETIVOS.}

El objetivo general es presentar un panorama de la situación de la investigación en las ciencias económicas, administrativas y contables de las instituciones de educación superior de Honduras.

Como objetivos específicos se presentan los siguientes:

- Conocer las tendencias de la educación superior en el área objeto de estudio, oferta, instituciones, matrícula, etc.

- Conocer y detallar la oferta de centros de investigación en el campo específico de las ciencias económicas, administrativas y contables.

- Enumerar las líneas o programas de investigación desarrolladas en las instituciones de educación superior en estas áreas del conocimiento.

- Conocer proyectos de investigación que están desarrollando los centros y unidades de investigación.

- Determinar el número de investigadores en estas ciencias.

- Conocer la producción científica en el área objeto de estudio, las publicaciones científicas, libros, manuales y documentos, los artículos científicos en revistas nacionales e internacionales indexadas.

- Evaluar la enseñanza de la investigación en los pensum de los grados académicos de las áreas del conocimiento atinentes.

- Conocer los programas o proyectos de investigación vinculados desde la academia con la empresa y gobierno.

\section{METODOLOGÍA.}

Para la elaboración de este trabajo, primeramente se realizó una revisión bibliográfica documental de información conceptual de la educación superior, específicamente a las tendencias de la docencia, investigación y vinculación en general y en las ciencias económicas, administrativas y contables. Otra actividad siguiente fue explorar antecedentes, orígenes, estadísticas, tendencias que se presentan en el mundo, la región latinoamericana y Honduras. 
Asimismo, se rescató información específicamente en la determinación de la oferta de instituciones, investigadores, líneas y programas, publicaciones, cobertura geográfica, vinculación docencia e investigación, investigación y vinculación.

Se diseñó una ficha de recolección de información general que explora en las instituciones de educación superior datos generales, de la oferta, infraestructura, recursos humanos, producción de los centros y programas de docencia y especialmente de investigación en las áreas económicas, administrativas y contables. También se realizaron algunas entrevistas para conocer de los principales actores involucrados en las instituciones de educación superior que realizan investigación en las áreas del conocimiento anteriormente señaladas, con el objetivo de ahondar en los aspectos cualitativos de centros, de sus programas, proyectos, líneas de investigación, fortalezas y debilidades de sus instituciones en la investigación económica, administrativa y contable, y conocer su visión a futuro.

\section{RESULTADOS.}

\subsection{La Educación Superior en Honduras.}

Durante la época colonial para fundar una Universidad se requería reunir una renta cuantiosa y permanente y además de contar con un número de personas competentes a fin de servir las cátedras, estos requerimientos hicieron casi imposible el surgimiento de instituciones de educación superior en el país. El país en esa época formaba parte de la Capitanía General de Guatemala y durante ese período llegaron a establecerse dos celebres universidades en la región, San Carlos en Guatemala en 1681 y León en Nicaragua, que de Seminario fundado en 1680 se convirtió en Universidad en 1814.

En Comayagua, Honduras se fundaron Cátedras de Gramática Latina en 1602, y en 1662 se fundó El Colegio San Agustín como Colegio Seminario. En 1733 fue inaugurado El Colegio Tridentino que marca el origen de la educación superior en el país. En el año de 1815 se presenta solicitud para convertir el Colegio Tridentino en Universidad y en 1820 para que se abriese las carreras de Leyes, Medicina y Mineralogía. En 1821 se propuso que fuese creado como universidad de segunda enseñanza, pero esos intentos no se cristalizaron. 
En los primeros años de la independencia se le dio al Colegio Tridentino una nueva organización y funcionó hasta 1827 cuando fue clausurado por la guerra civil. No es sino hasta el 14 de diciembre de 1845 que se inaugura en Tegucigalpa la Sociedad del Genio Emprendedor y Del Buen Gusto, siendo el rector el Presbítero José Trinidad Reyes. En el año de 1846 la Cámara de Representantes le da protección y en 1847 se solicita convertir a la sociedad en Universidad, alcanzando ese estatus el 19 de septiembre de 1847, siendo el nacimiento de la Universidad Nacional Autónoma de Honduras (UNAH), la primera en el país (Portillo Saenz, 1998)

La educación superior hondureña data desde el año de 1847, transcurriendo 130 años con solamente una institución de educación de ese nivel, no es sino hasta 1978 cuando se abren dos nuevos centros, en la década de los ochenta cuatro centros, en la década de los noventa seis centros y en la pasada última década siete centros para hacer un total de veinte centros hasta el 2011 (Tabla 1). En este devenir del tiempo se ha pasado de grandes campus urbanos a multi-campus que también son urbanos y están diseminados en las principales ciudades del país.

Tabla 1

Honduras: Centros de Educación Superior por creación, tipo, oferta y matrícula

\begin{tabular}{|r|l|c|c|c|r|r|}
\hline No. & Centros de Educación Superior & Siglas & $\begin{array}{c}\text { Año de } \\
\text { Creación }\end{array}$ & $\begin{array}{c}\text { Tipo de } \\
\text { CES }\end{array}$ & $\begin{array}{c}\text { Oferta de } \\
\text { grados } \\
2011\end{array}$ & $\begin{array}{c}\text { Matrícula } \\
\text { total 2011 }\end{array}$ \\
\hline 1 & $\begin{array}{l}\text { Universidad Nacional Autónoma } \\
\text { de Honduras }\end{array}$ & UNAH & 1847 & Pública & 123 & 70,303 \\
\hline 2 & $\begin{array}{l}\text { Universidad Pedagógica Nacional } \\
\text { Francisco Morazán }\end{array}$ & UPNFM & 1989 & Pública & 40 & 28,673 \\
\hline 3 & $\begin{array}{l}\text { Universidad Nacional de } \\
\text { Agricultura }\end{array}$ & UNA & 1994 & Pública & 6 & 2,122 \\
\hline 4 & $\begin{array}{l}\text { Escuela Nacional de Ciencias } \\
\text { Forestales }\end{array}$ & ESNACIFOR & 1994 & Pública & 4 & 149 \\
\hline 5 & $\begin{array}{l}\text { Universidad Nacional de la } \\
\text { Policía de Honduras }\end{array}$ & UNPH & 1996 & Pública & 6 & 664 \\
\hline 6 & $\begin{array}{l}\text { Universidad de la Defensa de } \\
\text { Honduras }\end{array}$ & UDH & 2005 & Pública & & 21 \\
\hline 7 & $\begin{array}{l}\text { Universidad José Cecilio del } \\
\text { Valle }\end{array}$ & UJCV & 1978 & Privada & & 891 \\
\hline 8 & Universidad de San Pedro Sula & USPS & 1978 & Privada & 16 & 5,216 \\
\hline 9 & $\begin{array}{l}\text { Universidad Tecnológica } \\
\text { Centroamericana }\end{array}$ & UNITEC & 1986 & Privada & 43 & 17,183 \\
\hline
\end{tabular}




\begin{tabular}{|c|c|c|c|c|c|c|}
\hline 10 & $\begin{array}{l}\text { Seminario Mayor Nuestra Señora } \\
\text { de Suyapa }\end{array}$ & SMNSS & 1986 & Privada & 3 & 167 \\
\hline 11 & $\begin{array}{l}\text { Universidad Tecnológica de } \\
\text { Honduras* }\end{array}$ & UTH & 1992 & Privada & 25 & 16,938 \\
\hline 12 & Escuela Agrícola Panamericana & EAP & 1988 & Privada & 4 & 1,218 \\
\hline 13 & $\begin{array}{l}\text { Universidad Católica de } \\
\text { Honduras }\end{array}$ & UNICAH & 1992 & Privada & 29 & 16,854 \\
\hline 14 & $\begin{array}{l}\text { Centro de Diseño, Arquitectura y } \\
\text { Construcción }\end{array}$ & CEDAC & 1996 & Privada & 4 & 227 \\
\hline 15 & $\begin{array}{l}\text { Universidad Cristiana Evangélica } \\
\text { Nuevo Milenio }\end{array}$ & UCENM & 2001 & Privada & 6 & 3,222 \\
\hline 16 & $\begin{array}{l}\text { Universidad Metropolitana de } \\
\text { Honduras }\end{array}$ & UMH & 2002 & Privada & 11 & 4,195 \\
\hline 17 & $\begin{array}{l}\text { Universidad Cristiana de } \\
\text { Honduras }\end{array}$ & UCRIS & 2004 & Privada & 7 & 1,054 \\
\hline 18 & $\begin{array}{l}\text { Instituto Superior Tecnológico } \\
\text { Jesús de Nazareth }\end{array}$ & USTJN & 2004 & Privada & 4 & 215 \\
\hline 19 & $\begin{array}{l}\text { Universidad Politécnica de } \\
\text { Honduras }\end{array}$ & UPH & 2005 & Privada & 4 & 976 \\
\hline 20 & $\begin{array}{l}\text { Universidad Politécnica de } \\
\text { Ingeniería }\end{array}$ & UPI & 2007 & Privada & 5 & 246 \\
\hline
\end{tabular}

Fuente: Elaboración propia en base a Estadísticas de Dirección de Educación

Superior de Honduras, 2008, 2011. *UTH matrícula preliminar.

E\&A

IIES

173

La educación superior hondureña en la última década ha alcanzado los niveles más grandes de su historia, es los últimos dieciséis años se incrementó el número de estudiantes matriculados en más de tres veces, actualmente el número de efectivos supera los 173,995 estudiantes (año 2011). Por modalidad, la presencial es la preponderante, ya que la modalidad virtual ha sido insignificante e incluso ha desaparecido del sistema, mientras que la educación a distancia ha aumentado su número y proporción, sin embargo, apenas supera un cuarto del total.

La oferta educativa de educación superior hasta el 2011 es de 363 grados, de los cuales la mitad de esta es ofrecida por instituciones públicas. El 55\% de la oferta de grados es ofrecida a nivel de licenciatura, siendo el grado académico de mayor oferta y aceptación estudiantil para realizar estudios, aun cuando no hay que olvidar que los grados superiores a las licenciaturas fueron ofertados más recientemente.

A través del tiempo ha existido una concentración espacial de la educación superior, primero en la ciudad capital y desde la década del sesenta se ubicó en las tres principales ciudades del país y no 
es sino hasta inicios de la década de los ochenta que se expandió a otras ciudades del occidente, sur, centro y oriente del país, primero en la oferta de centros de naturaleza pública y más recientemente por la proliferación de los de carácter privado, principalmente desde fines de los noventa e inicios del dos mil. Aun así, persiste una alta concentración educativa en las dos principales ciudades del país, que son Tegucigalpa y San Pedro Sula.

La educación superior publica en la matricula total es mayoritaria, con una tendencia a la disminución, en los últimos quince años ha disminuido su proporción del $87 \%$ al $60 \%$. En los matriculados de primer ingreso ha pasado de $81 \%$ al 51\% y en el año 2007 por primera vez la matricula de educación privada superó a la pública como un hito histórico, misma situación ocurrió en el 2009. En el 2010 la matrícula de educación pública y privada cada una absorbe la mitad de nuevos ingresos en las universidades hondureñas.

En las áreas del conocimiento en el 2010 hay una alta concentración de la oferta educativa de la educación superior en el sector de "ciencias sociales, ciencias comerciales y derecho", que sumadas a la enseñanza de la educación y humanidades alcanza el 66\%. Las ciencias y las ingenierías alcanzan solamente el $19 \%$, la salud el $12 \%$ y el resto agricultura y los servicios.

La cobertura educativa superior en la población en el país es baja, alcanza el 15.7\% y su avance a través del tiempo ha sido lento, desde el año de 1997 que era de 7\% transcurrieron 18 años para duplicarse y alcanzar el nivel actual (Flores, 2012).

\subsection{Las Ciencias Económicas, Administrativas y Contables en la Educación Superior en Honduras.}

Los estudiantes de educación terciaria a nivel mundial muestran un crecimiento sin precedentes, se ha quintuplicado su volumen, elevándose de 28,6 millones en 1970 a 152,5 millones en 2007. Hay una tendencia a la feminización en la matrícula. La mayoría de los países no reporta datos por campos de estudio, sin embargo, en 80 países se exhiben variaciones en participación en la educación superior y del tamaño de sus sectores terciarios. Aun así, los datos revelan que las Ciencias Sociales, Educación Comercial y Derecho se convierten en el primer campo seleccionado por los graduados. 
Según la UNESCO alrededor del año 2006, más de 14,5 millones de estudiantes estaban matriculados en la educación superior en la región Latinoamericana, con una alta concentración en ocho países e incluso una sola carrerea en casi todos los países (administración de empresas) concentraba un tercio del total de la matrícula. La demanda de estudios de posgrado en algunos países de América Latina se incrementó en los años noventa, principalmente en los programas de maestría por el alza en las ciencias sociales y administrativas, el área más grande de todas, que comprenden la administración de empresas, leyes, psicología, economía y ciencias sociales (CEPAL, 2009).

En el caso de Honduras, de la matrícula total de 169,878 estudiantes de la educación superior del 2010, estimamos unas 72 carreras del sector de ciencias comerciales que incluyen, técnicos, licenciaturas, maestrías, especialidades y doctorados, para un total de 51,450 estudiantes que representa $30.3 \%$ de la matrícula total de la educación superior de ese año (Tabla 2).

Catorce de veinte centros de educación superior ofrecen formación en esta área del conocimiento y solamente dos son de carácter publico, (pero que absorben el 41.2\% de matrícula, 22 carreras en tres niveles de grados, Técnico, Licenciatura y Maestría), por lo que esta área de formación es ofertada mayormente por los centros de educación privada (12), ya que la inversión en capital humano, equipos e infraestructura es menos costosa y altamente rentable en comparación con otras áreas de conocimiento.

Tabla 2

Honduras: Centros, Oferta y Matrícula de Ciencias

Comerciales en la Educación Superior, 2010

\begin{tabular}{|l|r|r|r|}
\hline \multicolumn{1}{|c|}{ Grado Universitario } & \multicolumn{1}{|c|}{$\begin{array}{c}\text { Centros de } \\
\text { Educación } \\
\text { Superior }\end{array}$} & $\begin{array}{l}\text { Oferta de } \\
\text { carreras }\end{array}$ & Matrícula Total \\
\hline Técnico Universitario & 4 & 8 & 739 \\
\hline Licenciatura & 14 & 33 & 47,137 \\
\hline Maestría & 6 & 30 & 3,511 \\
\hline Especialidad & 0 & 0 & 0 \\
\hline Doctorado & 1 & 1 & 63 \\
\hline Total* & 14 & 72 & 51,450 \\
\hline
\end{tabular}

*El total de Centros de Educación Superior que enseñan

Ciencias Comerciales son 14 de 20 existentes.

Fuente: Elaboración propia en base a Estadísticas de Educación Superior de Honduras, 2011. 
Las diez carreras con mayor matrícula total en la educación terciaria hondureña en el 2010 son: Administración de Empresas, Derecho, Medicina, Pedagogía, Mercadotecnia, Ingeniería Civil, Gerencia de Negocios, Contaduría Pública y Finanzas, Ingeniería Industrial y Psicología.

La carrera con mayor matrícula en la educación superior hondureña es "administración de Empresas", que tiene 11,456 estudiantes, $6.7 \%$ del total y el $22.3 \%$ del área de ciencias comerciales, que incluso en la realidad tiene un mayor número, si le agregan otras similares, con otros nombres, pero con formación similar, como Gerencia de Negocios (5,726 estudiantes) y otras administraciones aplicadas como: la industrial, agrícolas, turísticas, hoteleras, agropecuarias, bancarias, aduaneras, cooperativas, internacionales, ventas, producción, comercio, finanzas, mercadeo, etc. Que podría llevar a que las carreras de administración y afines alcancen una matrícula de 41,549 estudiantes, que representarían un $24.5 \%$ del total de CES y un $80.8 \%$ del total del área comercial, lo que indica su supremacía nacional en la educación terciaria hondureña y que sigue el mismo patrón latinoamericano y mundial de formación en está área del conocimiento.

En el caso de la Contaduría esta área del conocimiento comercial solo es ofrecida en dos grados, técnico y licenciatura, tres carreras y por tres universidades, una pública y dos privadas. Un total de 6,724 estudiantes que representa el esta área el $13.1 \%$ de la matrícula estudiantil en el área comercial.

Economía es la de menor matrícula en las ciencias comerciales, solo 975 estudiantes, principalmente en un centro público y a nivel de pregrado. Una carrera que si está en vías de extinción en Honduras es Administración Pública, que solamente cuenta con 221 estudiantes de pregrado.

\subsection{La investigación en las Ciencias Económicas, Administrativas y Contables en la Educación Superior.}

Las universidades públicas de la región de América Latina son las instituciones en las que se forman las personas y se realiza la mayor parte de la investigación y el desarrollo científico y tecnológico de cada país en las diferentes áreas del conocimiento, pero pocas son de alto nivel internacional.

Honduras no es la excepción, principalmente en la formación de estudiantes y también en la generación de la mayor parte de la investigación nacional de manera general, sin embargo, en las 
áreas comerciales la formación actualmente recae mayormente en los CES Privados, sin embargo, en el caso de la investigación, en esos centros de educación superior privados no es una prioridad fundamental, ya que no cuentan con centros de investigación, grupos de investigación o profesores investigadores con el quehacer principal y fundamental de investigar. Si se realiza investigación especialmente a nivel estudiantil, tanto a pregrado, pero con mayor énfasis a nivel de postgrado.

\subsubsection{Centros de Investigación.}

En la educación superior hondureña la investigación en las ciencias económicas administrativas y contables recae principalmente en los centros y programas de postgrados y en menor medida en los programas académicos de pregrado (Tabla 3).

Existe solo un centro dedicado a la investigación en el área, es el Instituto de Investigaciones Económicas y Sociales (IIES) en la Facultad de Ciencias Económicas de la UNAH, de carácter público que desde el año 1958 ha estado dedicado a través del tiempo a las labores investigativas, docencia y vinculación. Dedicados en su inicio a la investigación económica, agrícola, cooperativista y social, educación superior y demografía. Asimismo, a la docencia económica y de la metodología de investigación, como en la ejecución de los Centros de Estudio y Trabajo "CET", que eran laboratorios multidisciplinarios de investigación de tesis de grado, con el objetivo de solucionar problemas nacionales, regionales y locales. A través del tiempo, el IIES ha abierto el abanico de la investigación a las áreas administrativas, empresariales, tecnologías de la información, educación superior, producción, competitividad y el emprendedurismo.

Programas académicos de Postgrados son otros organismos especializados de investigación, en el área sobresale el Postgrado Centroamericano de Economía y Planificación del Desarrollo, (POSCAE) de la UNAH, creado en 1978, tiene la única Maestría de Economía en Honduras y en Centroamérica. Tal programa tiene como ejes transversales, la investigación científica, la teoría económica, la teoría del desarrollo y la política económica. Cuenta con una revista y un boletín de publicación regular, en los cuales se divulgan algunas de sus investigaciones. Las tesis de postgrado que elaboran sus egresados, son las más sólidas de su clase en el área económica. Las investigaciones terminadas 
sobre problemas económicos nacionales superan las 200 (Hernández, 2012). El POSCAE en sus 33 años ha dado paso a 17 promociones de estudiantes nacionales y extranjeros que hacen un total de 294 , correspondiendo el $64 \%$ a jóvenes hon $\neg$ dureños y un $34 \%$ ha correspondido a participantes de la región de C.A. y el Caribe (Martínez, 2012).

Tabla 3

Honduras: Institutos, Postgrados y Pregrados en las Ciencias Económicas, Administrativas y Contables en la Educación Superior

\begin{tabular}{|c|c|c|c|}
\hline $\begin{array}{l}\text { Centros de Educación } \\
\text { Superior }\end{array}$ & $\begin{array}{l}\text { Institutos de } \\
\text { investigación }\end{array}$ & Postgrados & Pregrado \\
\hline UNAH & $\begin{array}{l}\text { Instituto de } \\
\text { Investigaciones } \\
\text { Económicas y } \\
\text { Sociales (IIES) }\end{array}$ & $\begin{array}{l}\text {-Postgrado Centroamericano de } \\
\text { Economía y Planificación del } \\
\text { Desarrollo (POSCAE), Maestría } \\
\text { Economía, Gestión, formulación } \\
\text { de proyectos. } \\
\text {-Postgrados de la Facultad de } \\
\text { Ciencias Económicas } \\
\text { (POSFACE), Maestrías en } \\
\text { administración de empresas, } \\
\text { dirección de negocios } \\
\text { internacionales, planificación y } \\
\text { desarrollo de Turismo, } \\
\text { Mercadotecnia, metodología } \\
\text { investigación económica y social. } \\
\text { Doctorado en Dirección } \\
\text { Empresarial }\end{array}$ & $\begin{array}{l}\text {-Carreras y Unidades de la Facultad de } \\
\text { Ciencias Económicas (FCE-TEG). } \\
\text {-Carreras área económica administrativas } \\
\text { en Centros regionales, CURN, CURLA, } \\
\text { CUROC, CURO, CURVA, CURC. } \\
\text {-Carreras administración agropecuaria en } \\
\text { CASUED. }\end{array}$ \\
\hline UNA & & & $\begin{array}{l}\text {-Carrera de administración de empresas } \\
\text { agropecuarias }\end{array}$ \\
\hline UJCV & & & $\begin{array}{l}\text {-Carreras de área administración: } \\
\text { industriales, turísticas, agrícolas. }\end{array}$ \\
\hline USPS & & -Maestría admón. Empresas & -Careras área administración. \\
\hline UNITEC & & $\begin{array}{l}\text {-Maestrías áreas Dirección } \\
\text { Empresarial, admón. Proyectos, } \\
\text { mercadotecnia, recursos humanos, } \\
\text { finanzas. }\end{array}$ & $\begin{array}{l}\text { - Carreras administración industrial, } \\
\text { turística, mercadotecnia y negocios } \\
\text { internacionales, finanzas. }\end{array}$ \\
\hline UTH & & $\begin{array}{l}\text {-Maestría en Finanzas, admón. } \\
\text { transporte, dirección empresarial y } \\
\text { mercadeo, recursos humanos, } \\
\text { dirección financiera, dirección } \\
\text { empresarial y gestión turística, } \\
\text { gerencia de negocios. }\end{array}$ & $\begin{array}{l}\text {-Carreras de Gerencia de negocios, } \\
\text { relaciones industriales, mercadotecnia, } \\
\text { comercio y negocios internacionales, } \\
\text { ingeniería financiera, comercial y } \\
\text { producción industrial. }\end{array}$ \\
\hline EAP & & & Dpto. administración de negocios. \\
\hline UNICAH & & $\begin{array}{l}\text {-Maestrías área administración de } \\
\text { empresas, Economía y Finanzas, } \\
\text { gestión de proyectos, gestión de la } \\
\text { calidad, gerencia servicios salud. } \\
\text {-Doctorado ciencias admtvas. }\end{array}$ & $\begin{array}{l}\text {-Carreras Facultad de Ciencias } \\
\text { administrativas. }\end{array}$ \\
\hline UCENM & & & $\begin{array}{l}\text {-Carrera Admón. Empresas, } \\
\text { Mercadotecnia. }\end{array}$ \\
\hline
\end{tabular}




\begin{tabular}{|l|l|l|l|}
\hline UMH & & $\begin{array}{l}\text {-Maestría en gerencia de } \\
\text { mercadotecnia, ingeniería de } \\
\text { negocios, ingeniería económica y } \\
\text { financiera. }\end{array}$ & $\begin{array}{l}\text {-Carreras de ingeniería de negocios, } \\
\text { gestión y contaduría empresarial, } \\
\text { marketing y negocios internacionales. }\end{array}$ \\
\hline UCRISH & & & $\begin{array}{l}\text {-Carreras Administración de empresas, } \\
\text { mercadotecnia. }\end{array}$ \\
\hline ISTJN & & & -Carrera Gerencia e negocios. \\
\hline UPH & & & -Carrera Gerencia de negocios. \\
\hline UPI & & -Carrera de ingeniería financiera. \\
\hline
\end{tabular}

Fuente: Elaboración propia en base a Estadísticas de Educación Superior de Honduras, 2011 y otras fuentes.

Los Postgrados de la Facultad de Ciencias Económicas de la UNAH (POSFACE) ahora agrupan los programas de Maestría de Administración de Empresas, Maestría en Dirección de Negocios Internacionales, Maestría en Planificación y Desarrollo de Turismo, Maestría en Mercadotecnia con énfasis en Negocios Internacionales, Maestría en Metodología de la Investigación Económica y Social. Recién se iniciará un programa de Doctorado en Dirección Empresarial.

\subsubsection{Profesores Investigadores.}

En la educación superior de Honduras habían en el 2009 unos 7,664 docentes, de los cuales solo 3,821 trabajaban a tiempo completo, 424 a medio tiempo y 3,419, todos estos llevaban a estimar que 4,833 eran profesores a tiempo completo equivalente.

En el caso de investigadores está categoría es reconocida como tal solamente en la UNAH por la dedicación, producción o formación en investigación, aun cuando no solamente se dedique a la investigación. En el 2009 la Dirección de Investigación Científica de la UNAH presentó el Catalogo de Investigadores oficialmente registrados, en el cual se inscribieron 81 investigadores de las diferentes áreas del conocimiento, en el caso de la Ciencias Económicas se registraron 6 investigadores (https:// www.unah.edu.hn/?cat $=1316 \&$ fcats). Actualmente se está preparando el segundo Catalogo de Investigadores, en los cuales hay registrados 16 investigadores en el área de Ciencias Económicas, trabajando en los temas prioritarios de investigación de la UNAH (Tabla 4).

A su vez la Secretaría de Planificación (SEPLAN) del Gobierno de Honduras ha creado el Sistema de Información y Registro de los Investigadores de Honduras (SIRIH), que esta compuesto por personas 
del sector público, social y privado que llevan a cabo actividades relacionadas con la investigación y el desarrollo de la Ciencia y Tecnología en Honduras. El SIRIH registra 206 investigadores, de los cuales 8 están registrados en el área económica ${ }^{1}$ (.http://www.sirih.org/?cat=1000\&title=Directo$\underline{\text { rio\&lang=es). }}$

\section{Tabla 4}

UNAH: Investigadores área económica por tema prioritario y actividad, 2012

\begin{tabular}{|c|c|c|c|c|c|c|c|}
\hline Actividad & $\begin{array}{c}\text { Infraestructura } \\
\text { y desarrollo } \\
\text { territorial }\end{array}$ & $\begin{array}{l}\text { Pobreza e } \\
\text { inequidad }\end{array}$ & $\begin{array}{c}\text { Globalización, } \\
\text { productividad } \\
\text { y } \\
\text { competitividad }\end{array}$ & $\begin{array}{c}\text { Reforma } \\
\text { del } \\
\text { estado }\end{array}$ & $\begin{array}{c}\text { Conflictividad } \\
\text { política y } \\
\text { social }\end{array}$ & $\begin{array}{c}\text { Población, } \\
\text { necesidades } \\
\text { básica y } \\
\text { transición } \\
\text { demográfica }\end{array}$ & $\begin{array}{l}\text { Educación } \\
\text { y cultura }\end{array}$ \\
\hline Formación & 1 & 4 & 3 & 1 & & 1 & 1 \\
\hline Capacitación & 2 & 2 & 1 & & 1 & 3 & 6 \\
\hline Enseñanza & & 1 & & & & & 3 \\
\hline $\begin{array}{l}\text { Capacitación } \\
\text { y enseñanza }\end{array}$ & & 2 & 1 & 1 & & & \\
\hline $\begin{array}{l}\text { Formación y } \\
\text { enseñanza }\end{array}$ & & & 1 & & & & \\
\hline $\begin{array}{l}\text { Formación, } \\
\text { capacitación } \\
\text { y enseñanza }\end{array}$ & 1 & & 3 & & & 1 & 1 \\
\hline Sin dato & 12 & 7 & 7 & 14 & 15 & 11 & 7 \\
\hline Total & 16 & 16 & 16 & 16 & 16 & 16 & 16 \\
\hline
\end{tabular}

Fuente: Elaborado con datos preliminares del Depto. de Políticas,

Dirección de Investigación Científica de la UNAH, 2012.

\subsubsection{Política de investigación.}

La Universidad Nacional Autónoma de Honduras (UNAH), es la institución de educación superior hondureña que mediante el programa de la Cuarta Reforma Universitaria, plantea "la promoción e Inversión en investigación, gestión del conocimiento y propuestas de solución a los problemas nacionales". En tal sentido, junto a la realización de la actividad científica se desarrollan competencias que contribuyen a la investigación dentro de las unidades académicas de la UNAH, para lo cual se

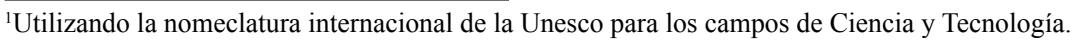


ha creado una política institucional que brinda las directrices en materia de investigación, desarrollo tecnológico e innovación para el quinquenio 2012-2016 (UNAH, 2011).

La política de investigación se concibe como un instrumento de ordenamiento de los esfuerzos y apoyo a la Investigación, Desarrollo e Innovación, el fortalecimiento institucional en su función de investigación, y la contribución al desarrollo de la ciencia y tecnología, y a la solución de los problemas nacionales y regionales, favoreciendo la cooperación e intercambio de activos intangibles a nivel interinstitucional, nacional e internacional, en materia de ciencia y tecnología e innovación. La define como el conjunto de objetivos, ejes, estrategias y acciones que guían la acción colectiva en materia de investigación, desarrollo tecnológico e innovación en las distintas unidades académicas. Se basa en dos ámbitos interno y externo, en el primero en la UNAH se consideran las carreras de grado y postgrado, los institutos de investigación, las unidades de investigación, las coordinaciones regionales de investigación, los grupos y redes de investigación, y los observatorios especializados adscritos a carreras de grado y posgrado o a los institutos de investigación. Todos ellos se articulan en los Consejos de Investigación de la facultad o del centro regional. En el externo, a nivel nacional, regional e internacional. Los ejes orientadores de la política de investigación son: el fomento, la publicación, difusión y comunicación, la protección de resultados, la capacitación y la gestión. La UNAH clasifica la investigación en cuatro grandes áreas: Investigación científica, tecnológica, artística y humanística. En cuanto al financiamiento de la política plantea alcanzar en el año 2016 el $2 \%$ del presupuesto de la institución.

La Facultad de Ciencias Económicas de la UNAH es la única unidad académica que ha conformado su Consejo de Investigación, reglamentado y conformado por la Decana de la Facultad, el Coordinador de los Posgrados de la Facultad, el Director del IIES, dos profesores investigadores del IIES y otros miembros de las diferentes unidades investigación de las carreras.

\subsubsection{Prioridades, ejes y temas prioritarios}

La UNAH, institución en la vanguardia de la investigación científica en Honduras tiene establecidos las prioridades de investigación para el quinquenio 2012-2016 en 13 temas agrupados en 4 ejes de investigación, siendo estos: Desarrollo económico y social, democracia y gobernabilidad, población y condiciones de vida y el de ambiente, biodiversidad y desarrollo. 
El eje "desarrollo económico y social", que es atinente al área de estudio de este trabajo, se entiende como la capacidad del país o región para generar condiciones de vida para los grupos o individuos de manera racional, en los cuales todos los elementos del proceso: económicos, tecnológicos y de conservación y utilización ecológica, están en correspondencia con la premisa del tipo de desarrollo que se quiere lograr, siendo el ser humano el centro del mismo. Tiene tres temas prioritarios con la inclusión de aspectos (Tabla 5).

\section{Tabla 5}

UNAH: Prioridades por Ejes, Temas y Aspectos de Investigación en las Ciencias Económicas, Administrativas y Contables.

\begin{tabular}{|c|c|c|}
\hline Eje & Tema & Aspectos \\
\hline \multirow[t]{3}{*}{$\begin{array}{l}\text { Desarrollo } \\
\text { económico y } \\
\text { social }\end{array}$} & $\begin{array}{l}\text { 1.Infraestructura y } \\
\text { desarrollo territorial }\end{array}$ & $\begin{array}{l}\text { 1. Economía de escala y de ámbito. } \\
\text { 2. Convergencia y cohesión económica, social y territorial. } \\
\text { 3. Articulación del territorio hacia adentro y hacia afuera. } \\
\text { 4. Difusión de la información y gestión del conocimiento mediante redes de } \\
\text { TV, sistemas de cable, estaciones de repetición, redes de suministro de } \\
\text { internet y otras. } \\
\text { 5. Ventajas competitivas asociadas al acceso a mercados y los costos de los } \\
\text { insumos. } \\
\text { 6. Desarrollo social asociado a la construcción de hospitales y escuelas. } \\
\text { 7. Cobertura y calidad de los servicios básicos vinculados al abastecimiento } \\
\text { de agua potable y saneamiento, electricidad, telecomunicaciones y } \\
\text { transporte colectivo intra - urbano e inter-urbano. }\end{array}$ \\
\hline & $\begin{array}{l}\text { 2.Pobreza e } \\
\text { inequidad }\end{array}$ & $\begin{array}{l}\text { 1. Determinantes de la pobreza y la inequidad. } \\
\text { 2. Nuevas propuestas metodológicas de medición de la pobreza y la } \\
\text { inequidad. } \\
\text { 3. Políticas públicas de combate a la pobreza y la inequidad (evaluación de } \\
\text { impacto). } \\
\text { 4. Riqueza cultural y natural versus pobreza e inequidad territorial. } \\
\text { 5. Servicios básicos, pobreza e inequidad. } \\
\text { 6. Pobreza, inequidad y costo social de la ineficiencia y corrupción pública. } \\
\text { 7. Grupos vulnerables, pobreza e inequidad. }\end{array}$ \\
\hline & $\begin{array}{l}\text { 3.Globalización, } \\
\text { productividad y } \\
\text { competitividad }\end{array}$ & $\begin{array}{l}\text { 1. Globalización: impacto y desafíos. } \\
\text { 2. Productividad y competitividad. } \\
\text { 3. Mercado interno y externo. } \\
\text { 4. Crisis económica y financiera. } \\
\text { 5. Innovación y tecnología. } \\
\text { 6. Cultura productiva y gestión empresarial. } \\
\text { 7. Turismo sostenible y gestión y conservación del patrimonio (cultural y } \\
\text { natural). } \\
\text { 8. Cadenas productivas y de valor. }\end{array}$ \\
\hline
\end{tabular}

Fuente: Prioridades de investigación UNAH 2012-2016, Ejes y Temas prioritarios, DICU-UNAH. 


\subsubsection{Líneas, programas y proyectos de investigación}

En el área de investigación de la universidad más grande y de mayor trayectoria investigativa del país, hay un proceso de reforma que involucra la mejora de la organización, de la planificación, de la administración y de la ejecución de la investigación. Por está razón, en el caso de las Ciencias Económicas, Administrativas y Contables, presentamos las líneas, proyectos, actores involucrados y principales actividades que fue propuesta por el IIES al Consejo de Investigación de la Facultad de Ciencias Económicas de la UNAH, que se están programando y desarrollando (Tabla 6).

Tabla 6

UNAH-FCE: Líneas, proyectos, Investigadores, Programas y Actividades en las Ciencias Económicas, Administrativas y Contables.

\begin{tabular}{|c|c|c|c|c|}
\hline Línea & Proyectos & Investigadores & $\begin{array}{l}\text { Programa de } \\
\text { POSFACE } \\
\text { sustentador de } \\
\text { línea }\end{array}$ & $\begin{array}{l}\text { Actividades } \\
\text { vinculación/resultados de } \\
\text { la línea }\end{array}$ \\
\hline $\begin{array}{l}\text { Competitividad y } \\
\text { productividad }\end{array}$ & $\begin{array}{l}\text { Producción de Alto } \\
\text { Rendimiento-HPM }\end{array}$ & IIES y POSFACE & $\begin{array}{l}\text { Doctorado y } \\
\text { Maestrías }\end{array}$ & $\begin{array}{l}\text { Primer, segundo y cuarto } \\
\text { sectores/transferencia de } \\
\text { conocimiento de la } \\
\text { competitividad y } \\
\text { productividad, } \\
\text { considerando la } \\
\text { producción del alto } \\
\text { rendimiento/clase mundial }\end{array}$ \\
\hline $\begin{array}{l}\text { Educación y } \\
\text { Economía }\end{array}$ & $\begin{array}{l}\text { Oferta y Demanda de } \\
\text { ES: Sistema } \\
\text { integrado de valor y } \\
\text { de suministro }\end{array}$ & $\begin{array}{l}\text { IIES, POSFACE y Redes } \\
\text { piloto Regionales UNAH }\end{array}$ & $\begin{array}{l}\text { Doctorado y } \\
\text { Maestrías }\end{array}$ & $\begin{array}{l}\text { Todos los sectores de la } \\
\text { sociedad/transferencia de } \\
\text { conocimiento sobre las } \\
\text { cadenas de valor y de } \\
\text { suministro de la ES }\end{array}$ \\
\hline $\begin{array}{l}\text { Innovación, } \\
\text { Incubación, } \\
\text { Aceleración y } \\
\text { Desarrollo }\end{array}$ & $\begin{array}{l}\text { Centro de Promoción } \\
\text { de Innovación y } \\
\text { Desarrollo (CPID): } \\
\text { Incubadoras de } \\
\text { Ciencia y de } \\
\text { Emprendimiento }\end{array}$ & $\begin{array}{l}\text { IIES, POSFACE y FCE } \\
\text { (Centro de } \\
\text { Emprendedores) }\end{array}$ & $\begin{array}{l}\text { Doctorado y } \\
\text { Maestrías }\end{array}$ & $\begin{array}{l}\text { Todos los sectores / } \\
\text { transferencia de } \\
\text { conocimiento por } \\
\text { incubadoras de ciencia y } \\
\text { de emprendimiento, } \\
\text { aceleradores y } \\
\text { desarrolladores como } \\
\text { centros de innovación. } \\
\text { semillero de } \\
\text { investigaciones e } \\
\text { investigadores }\end{array}$ \\
\hline $\begin{array}{l}\text { Economía y } \\
\text { Emprendimiento }\end{array}$ & $\begin{array}{l}\text { Observatorio } \\
\text { Económico y de } \\
\text { Emprendimiento } \\
\text { (OEE) }\end{array}$ & IIES y POSFACE & $\begin{array}{l}\text { Doctorado y } \\
\text { Maestrías }\end{array}$ & $\begin{array}{l}\text { Todos los sectores. Acceso } \\
\text { base de datos de } \\
\text { Observatorio, tanque de } \\
\text { pensamiento de economía } \\
\text { y emprendimiento que } \\
\text { apoye académicos, } \\
\text { profesionales y tomadores } \\
\text { de decisión }\end{array}$ \\
\hline
\end{tabular}

Fuente: Ortega, César. Formación Investigadora, programas y Proyectos en Líneas de Investigación, Propuesta del Instituto de Investigaciones Económicas y Sociales para el Consejo de Investigación de la Facultad de Ciencias Económicas de la UNAH, Tegucigalpa, IIES, 2011. 
En el caso de los proyectos de investigación a nivel de la educación superior las estadísticas de estos, específicamente en las ciencias económicas administrativas y contables no han recogido exhaustivamente los proyectos de investigación en la mayoría de los centros, ya que son muy pocos, y en otros casos más bien ha estado sobredimensionado, posiblemente porque se incluyeron la elaboración de trabajos o tesis de grado (Tabla 7).

\section{Tabla 7}

Honduras: Proyectos de Investigación en área Económica, administrativa y Contable según Centro de Educación Superior, 2006-2010

\begin{tabular}{|r|l|c|c|c|c|c|}
\hline No. & \multicolumn{1}{|c|}{ Centros de Educación Superior } & 2006 & 2007 & 2008 & 2009 & 2010 \\
\hline 1 & Universidad Nacional Autónoma de Honduras & $\mathrm{n}$ & 2 & 2 & $\mathrm{n}$ & 1 \\
\hline 2 & $\begin{array}{l}\text { Universidad Pedagógica Nacional Francisco } \\
\text { Morazán }\end{array}$ & $\mathrm{n}$ & $\mathrm{n}$ & $\mathrm{n}$ & $\mathrm{m}$ & $\mathrm{m}$ \\
\hline 3 & Universidad Nacional de Agricultura & $\mathrm{a}$ & $\mathrm{a}$ & $\mathrm{a}$ & $\mathrm{a}$ & $\mathrm{a}$ \\
\hline 4 & Escuela Nacional de Ciencias Forestales & $\mathrm{a}$ & $\mathrm{a}$ & $\mathrm{a}$ & $\mathrm{a}$ & $\mathrm{a}$ \\
\hline 5 & $\begin{array}{l}\text { Universidad Nacional de la Policía de } \\
\text { Honduras }\end{array}$ & $\mathrm{a}$ & $\mathrm{a}$ & $\mathrm{a}$ & $\mathrm{a}$ & $\mathrm{a}$ \\
\hline 6 & Universidad de la Defensa de Honduras & 9 & 9 & 3 & 3 & 14 \\
\hline 7 & Universidad José Cecilio del Valle & $\mathrm{m}$ & $\mathrm{m}$ & $\mathrm{n}$ & $\mathrm{n}$ & 1 \\
\hline 8 & Universidad de San Pedro Sula & 2 & 1 & 1 & 2 & 2 \\
\hline 9 & Universidad Tecnológica Centroamericana & $\mathrm{m}$ & $\mathrm{m}$ & $\mathrm{m}$ & $\mathrm{n}$ & $\mathrm{n}$ \\
\hline 10 & Seminario Mayor Nuestra Señora de Suyapa & $\mathrm{a}$ & $\mathrm{a}$ & $\mathrm{a}$ & $\mathrm{a}$ & $\mathrm{a}$ \\
\hline 11 & Universidad Tecnológica de Honduras & $\mathrm{m}$ & $\mathrm{m}$ & 250 & 250 & $\mathrm{~m}$ \\
\hline 12 & Escuela Agrícola Panamericana & $\mathrm{a}$ & $\mathrm{a}$ & $\mathrm{a}$ & $\mathrm{a}$ & $\mathrm{a}$ \\
\hline 13 & Universidad Católica de Honduras & $\mathrm{m}$ & $\mathrm{m}$ & $\mathrm{m}$ & 56 & 56 \\
\hline 14 & Centro de Diseño, Arquitectura y & $\mathrm{a}$ & $\mathrm{m}$ & $\mathrm{m}$ & $\mathrm{m}$ & $\mathrm{a}$ \\
\hline 15 & Universidad Cristiana Evangélica Nuevo & $\mathrm{m}$ & 1 & $\mathrm{n}$ & $\mathrm{n}$ & $\mathrm{n}$ \\
\hline 16 & Universidad Metropolitana de Honduras & $\mathrm{m}$ & $\mathrm{m}$ & 3 & $\mathrm{~m}$ & 3 \\
\hline 17 & Universidad Cristiana de Honduras & $\mathrm{m}$ & 6 & 4 & 16 & 15 \\
\hline 18 & $\begin{array}{l}\text { Instituto Superior Tecnológico Jesús de } \\
\text { Nazareth }\end{array}$ & & & 3 & 8 & $\mathrm{~m}$ \\
\hline 19 & Universidad Politécnica de Honduras & $\mathrm{n}$ & $\mathrm{a}$ & $\mathrm{n}$ & 1 & 16 \\
\hline 20 & Universidad Politécnica de Ingeniería & 9 & $\mathrm{n}$ & 12 & $\mathrm{n}$ \\
\hline
\end{tabular}

Fuente: Elaboración propia en base a Estadísticas de Dirección de Educación Superior de Honduras, 2006-2010.

a: Categoría sin objeto.

m: Datos no disponibles.

n: Cifras nula

\subsubsection{Resultados de Investigación.}

Los resultados de investigación en las áreas de conocimiento en estudio se hacen a través de la 
producción de informes de investigación, tesis de grado, especialmente de postgrados, revistas y boletines específicos. En el caso del IIES de la UNAH tiene dos órganos de difusión científica "Revista Economía Política" actualmente "Revista Economía y Administración” y un Boletín del IIES, ambos indexados (http://www.iies-unah.org/). En el caso del POSCAE tiene una Revista de Economía y el boletín Análisis Económico. Otras universidades carecen de revistas de contenido científico académico, si producen tesis de grado con diferente grado de rigurosidad.

\subsubsection{Publicaciones.}

Para conocer el perfil detallado de la actividad investigadora de las Instituciones de Educación Superior se elabora el Ranking Iberoamericano SIR, basándose en datos cuantitativos de publicación y citación. Para su elaboración en el 2012 se ha analizado toda la producción científica presente en la base de datos Scopus, elaborada por Elsevier, en el periodo 2006-2010 y se ha asociado cada publicación y cada cita encontrada a la institución o instituciones correspondientes ${ }^{2}$.

En el caso de Honduras, solo 7 instituciones de educación superior aparecen en el ranking ${ }^{3}$, ninguna en el rango de las primeras 100, ni las primeras 250, una en las primeras 500, 2 en las primeras 1,000 y 7 en el total de las 1,401 universidades. La UNAH el primer lugar de actividad investigativa de Honduras, sin embargo ocupa el lugar 498 en el ranking iberoamericano y el 399 en el ranking Latinoamericano y el Caribe. Los indicadores muestran 70 publicaciones en revistas científicas (PC), 87.1 es el ratio de colaboración internacional (CI), 0.7 es la calidad científica promedio (CCP), 48.6 es el porcentaje de publicación en revistas del primer cuartil (Q1) y 9.0 es ratio de excelencia (ER).

La base de datos SCImago Journal and Country Rank de los años 1996-2010 ubica a Honduras en el lugar 150 de 236 países, con 520 documentos, 508 documentos citados, 5,381 citas, 300 mismas citas, 11.68 citas por documento y $34 \mathrm{H}$ index, todo esto de todas las áreas del conocimiento, donde sobresalen principalmente Medicina, agricultura y ciencias biológicas, inmunología y microbiología.

\footnotetext{
${ }^{2}$ El ranking muestra cinco indicadores: producción científica, ratio de colaboración internacional, calidad científica promedio de cada institución, proporción de artículos publicados en revistas de prestigio y ratio de excelencia investigadora (definición más precisa en la barra lateral). Además se incluyen las tasas de variación de cada uno de los valores con respecto al valor del periodo 2005-2009.

${ }^{3}$ 1. UNAH en posición IBE 498 con 70 publicaciones, 2. EAP en posición 617 con 38 publicaciones, 3. ESNACIFOR en posición 1020 con 5 publicaciones, 4. UPNFM en posición 1044 con 5 publicaciones, 5. UNA en posición 1160 con 3 publicaciones, 6. UNICAH en posición 1,219 con 2 publicaciones, 7. UNITEC en posición 1,383 con 1 publicaciones.
} 
Tabla 8

Honduras: Publicaciones en Revistas Científicas, 1996-2010

Negocios, Gerencia y Contabilidad y Economía, Econometría y Finanzas)

\begin{tabular}{|c|c|c|c|c|c|}
\hline Años & $\begin{array}{l}\text { Negocios y } \\
\text { Gerencia } \\
\text { Internacional }\end{array}$ & $\begin{array}{l}\text { Gerencia de } \\
\text { Tecnología e } \\
\text { Innovación }\end{array}$ & $\begin{array}{l}\text { Es trategia y } \\
\text { Gerencia }\end{array}$ & $\begin{array}{l}\text { Economía y } \\
\text { Econometría }\end{array}$ & Total \\
\hline 1996 & - & - & - & - & - \\
\hline 1997 & - & - & 1 & 1 & 2 \\
\hline 1998 & - & - & - & - & - \\
\hline 1999 & 1 & 1 & - & - & 2 \\
\hline 2000 & - & - & - & 1 & 1 \\
\hline 2001 & - & - & - & 1 & 1 \\
\hline 2002 & - & - & - & 1 & 1 \\
\hline 2003 & - & - & - & 1 & 1 \\
\hline 2004 & - & - & - & 1 & 1 \\
\hline 2005 & - & - & - & - & - \\
\hline 2006 & - & - & - & - & 1 \\
\hline 2007 & - & - & - & 1 & 1 \\
\hline 2008 & - & - & - & 1 & - \\
\hline 2009 & - & - & - & - & - \\
\hline 2010 & - & - & - & - & \\
\hline
\end{tabular}

Fuente: Elaboración propia en base de datos SCImago, www.scimagojr.com

Ahora en las áreas de conocimiento comerciales, hay dos áreas la de negocios, gerencia y contabilidad y la otra de economía, econometría y finanzas. En la primera área, Honduras se ubica en el número 146 de 180 países, con 3 documentos, 3 documentos citados, 4 citas, 0 mismas citas, 1.5 citas por documento y $2 \mathrm{H}$ index. En el área de economía, ecometría y finanzas, el país tiene el lugar 120 de 188 países, con 8 documentos, 8 documentos citados, 100 citas, 1 misma cita, 12.50 citas por documento y $4 \mathrm{H}$ index. De plano hay una mayor producción científica en el área económica en comparación con la administrativa contable, sin embargo, es insignificante comparada con otras áreas del conocimiento y con otros países de la región Centroamericana, como Costa Rica y El Salvador (Tabla No.8).

\subsubsection{La Investigación en los procesos de enseñanza en la Educación Superior.}

La investigación científica en Honduras necesita una mayor articulación investigación-docencia. 
Ello se aplica no sólo a la propia Facultad de Ciencias Económicas de la UNAH, sino también a la Universidad misma y a la Educación Superior del país en general. Dicha integración de la investigación permitirá facilitar la formación investigadora de docentes y estudiantes, y una mayor vinculación de la investigación científica con el entorno social. En este sentido, se describe brevemente algunos puntos importantes que deben ser tratados para lograr dicha articulación.

Existencia de muy pocos docentes a tiempo completo con dedicación exclusiva, y una reducida cantidad de doctores. Reducido apoyo de la administración a las actividades de investigación, la mayoría de los recursos los absorbe la docencia universitaria. No existe ningún método de acreditación objetivo que permita medir y diferenciar el desempeño de profesores y también del profesor-investigador. Ello impide agrupar a los profesores según sus competencias para una asignación de tareas acorde a sus conocimientos.

En la Facultad de Ciencias Económicas de la UNAH todas las carreras tienen contemplado en su pensum de estudio la asignatura de "Métodos y Técnicas de Investigación" y la carrera de Economía es la responsable por la organización y enseñanza. Para el año 2010, se matricularon 1,350 estudiantes en diez secciones y el proceso de enseñanza estuvo a cargo de 10 docentes, 9 estaban a tiempo completo y 1 por hora; 1 tiene doctorado, 6 con maestría, 1 pasante de maestría y el resto nivel de licenciatura. Algunos docentes tienen publicaciones de artículos y documentos, la mayoría publicados para las instituciones en las que trabajan (Barahona, 2012).

En la UNAH con la creación en los últimos años de la Maestría en Metodologías de Investigación Económica y Social se ha abierto un semillero de jóvenes profesionales formados en la investigación, muchos de los cuales serán o son los profesores de las facultades atinentes y que han aumentado sus habilidades investigativas útiles en la enseñanza universitaria.

\subsubsection{La investigación en la vinculación academia, empresa y gobierno.}

En el caso de la vinculación de centros públicos y privados con el gobierno, ya hay una mayor apertura en los últimos años, principalmente en aquellos rubros donde el conocimiento científico universitario incide o colabora con su expertise en algunas áreas del conocimiento, principalmente en el caso 
de las universidades públicas, donde el estado ha hecho inversiones. Hay muy poca vinculación entre la academia y la empresa y entre la academia y el gobierno, aunque esta afirmación no es una generalidad, ya que en los últimos años hay un mayor acercamiento entre los centro de educación superior privados con las empresas, en relación a los centros públicos. La vinculación de la academia, con la empresa y el gobierno es una tarea a desarrollar en el país, principalmente en la utilización de generación de conocimiento científico en el área económica, administrativa y contable.

Un proyecto de investigación "oferta y Demanda de la Educación Superior en Honduras: Cadena de valor y suministro", que desarrolla el IIES puede arrojar luces en varios aspectos de la educación terciaria, entre ellos el desajuste de la oferta y la demanda de profesionales y de proyectos de investigación, así como de la vinculación entre los empleadores, los graduados, la academia y los suministros de ella.

\section{CONCLUSIONES.}

1.- La matrícula de la educación superior ha alcanzado los niveles más grandes de su historia, es los últimos dieciséis años se incrementaron los estudiantes en más de tres veces, actualmente supera los ciento setenta mil estudiantes, sin embargo, la cobertura educativa del nivel es baja (16\%).

2.- En las áreas del conocimiento actualmente hay una alta concentración de la oferta educativa en el sector de ciencias sociales, ciencias comerciales y derecho, que sumadas a la enseñanza de la educación y humanidades alcanza los dos tercios del total, catorce de veinte centros tienen en su oferta su formación.

3.- La carrera de administración y afines tiene supremacía nacional en la educación terciaria hondureña, siguiendo las tendencias regionales y mundiales de formación en está área del conocimiento, representa un cuarto del total y el $80.8 \%$ del total del área comercial.

4.-.La investigación de la educación superior en el país está concentrada en las instituciones públicas. En las ciencias económicas, administrativas y contables también se reproduce el patrón de primacía en las instituciones públicas. 
5.- Solo una institución de educación superior tiene una política de investigación, con ejes y temas prioritarios, infraestructura organizativa, un centro especializado en investigación del campo, postgrados con producción científica y la única Maestría en Metodologías de Investigación Económica y Social.

6.- El mayor número de investigadores en la región, se ubican mayoritariamente en las instituciones de educación superior, sobre todo en las universidades públicas y en Honduras no es la excepción.

7.- La producción científica en las ciencias comerciales (economía, administración y contaduría) de las instituciones de educación superior es muy baja, con respecto a otras áreas del conocimiento, aun cuando ofrece la mayor oferta de estudios.

8.- La enseñanza de la investigación en la educación superior está mejor estructurada en los programas de postgrados.

9.- Existe poca vinculación entre la academia, el gobierno y las empresas. 


\section{REFERENCIAS}

- Barahona, Blanca Celea (2012). La Enseñanza de la Metodología de la Investigación Científica en la Facultad de Ciencias Económicas de la UNAH Diagnóstico y Propuesta. 2009-2011. Tesis de Maestría en Metodología de la Investigación Económica y Social, Tegucigalpa, UNAH.

- CEPAL, (2009). La Educación superior y el desarrollo económico en América Latina, Serie Estudios y Perspectivas No.106, México, CEPAL.

- DES (2008, 2009, 2010, 2011). Anuario Estadístico del Nivel de Educación Superior, 2007, 2008, 2009, 2010. Tegucigalpa, Dirección de Educación Superior.

- Flores Fonseca, Manuel (2012). Tendencias de la Educación Superior en Honduras, Tegucigalpa, IIES-UNAH. (borrador).

- Hernández Martínez, Lisandro (2012). Vigencia de la carrera de Economía y las nuevas generaciones de universitarios, Análisis Económico, año 17, No.42, mayo 2012. Tegucigalpa, POSCAE, UNAH.

- Martínez, José Luis (2012). ¿Porqué estudiar Economía?, Análisis Económico, año 17, No.42, mayo 2012. Tegucigalpa, POSCAE, UNAH.

- Ortega, César (2011). Formación Investigadora, programas y Proyectos en Líneas de Investigación, Propuesta del Instituto de Investigaciones Económicas y Sociales para el Consejo de Investigación de la Facultad de Ciencias Económicas de la UNAH, Tegucigalpa, IIES. 
- Portillo Saenz, A. (1988). La Educación Superior en Honduras 1733-1997, Vol.II. Tegucigalpa, SCANCOLOR.

- UNAH (2011). Política de Investigación Científica, Desarrollo Tecnológico e Innovación, I+D+I, 2002-2016. Tegucigalpa. UNAH.

- UNESCO-UIS (2006a). Compendio Mundial de Educación 2006: Comparación de las estadísticas de educación en el mundo. Montreal: Instituto de Estadísticas de la Organización de las Naciones Unidas para la Educación, la Ciencia y la Cultura.

- UNESCO-UIS (2009). Compendio Mundial de la Educación 2009, Comparación de las estadísticas de educación en el mundo. Montreal, Instituto de Estadística de la Organización de las Naciones Unidas para la Educación, la Ciencia y la Cultura. 